\title{
OPTIMIERUNG DES DIDAKTISCHEN PROZESSES UND STREBEN NACH SUBJEKTIVITÄT. „OFFENER" GEIST
}

\author{
KATARZYNA IŻBIN̉SKA \\ Adam Mickiewicz University - Poznań
}

\begin{abstract}
ABSTRACr. The following article discusses the optimization of the didactic process by using some relevant elements and by simultaneous aiming at subjectivity. Moreover, the new role of the teacher, who also becomes the mentor of his students, contributes to the improvement of this didactic process.

The author of this article claims that the whole pedagogical process is the system of multiple elements which stay in mutual correlation. Not taking under consideration, explained in the article, components or isolating them from each other may lead to disturbances in functioning of the system or even its collapse which means the destruction of the didactic interaction.
\end{abstract}

Der didaktische Prozess bedarf im Informationszeitalter des 20. Jahrhunderts einer Veränderung der Form. Sein bisheriger Charakter erfüllt kaum die Erwartungen der Lerner, was in vielen Fällen Resignation, Lernapathie, Demotivation und immer öfter Flucht der jungen Menschen in die Welt der Drogenund Alkoholsucht zur Folge hat. Die Menschen werden jeden Tag mit vielen Informationen überflutet und sind oft nicht im Stande im Informationschaos das Richtige auszuwählen. Viele können ihre eigenen Ziele nicht definieren, ohne dabei eine Vision der Zukunft zu haben. Das traditionelle Schulsystem ist auf den Enzyklopädismus und die allgemeine Vermittlung von moralischen Werten ohne Berücksichtigung einer Individualisierung konzentriert. Der permanente Fortschritt in allen Lebensbereichen erfordert eine Steigerung der beruflichen Kompetenz, was mit einer Flexibilisierung des Bildungsmodells und einer ständigen Verbesserung seines Niveaus zusammen vorkommen soll. Die immer mehr steigende Konkurrenz ist auf eine dynamische sowie politik- und wirtschaftsbedingte Entwicklung Polens zurückzuführen. In der Schule des 21. Jahrhunderts soll nicht das Merken, sondern das Denken, und nicht die passive Rekonstruierung eines Inhalts, sondern die Kreativität und die intellektuelle Selbständigkeit entwickelt werden. $\mathrm{Zu}$ den Aufgaben dieser Schule soll auch Folgendes gehören: Entwicklung von Selbstwertgefühl und Individualität, Flexibilisierung und Bereitschaft zur ständigen Vornahme von Änderungen. Die 
Lerner müssen über die Subjektivität verfügen. Sie sollen im Mittelpunkt des didaktischen Prozesses stehen. Der Lehrer dagegen soll die Rolle eines Führenden in der Welt der Wissenschaft spielen.

Die Schule soll den Menschen mit „offenem" Geist ausstatten. Nur ein solcher Geist vermag in der sich ständig wandelnden Welt entsprechend zu handeln und in die Avantgarde seiner Generation zu gelangen.

Im Informationszeitalter steht die Kommunikation im Zentrum und mit ihrer exponierten Stellung wächst die Rolle der Fremdsprachen in der Welt. Das fossilisierte Bildungssystem bedarf vieler Veränderungen. Viele Lerner haben negative Assoziationen mit der Schule. Die Ausschreitungen der jungen Menschen signalisieren, dass Veränderungen dringend vorzunehmen sind.

Ich ließ mich bei dieser Problematik von zwei Quellen inspirieren: der aktuelle alarmierende Sachinhalt in diesem Bereich und die von Frau Prof. Prokop präsentierte alternative Lernmethode - Suggestopädie. Meine Teilnahme an den Lehrveranstaltungen von Frau Prof. Prokop am Lehrstuhl für Glottodidaktik und Translatorik im Diplomstudium und im Tagesstudium im Rahmen der Fakultät Sprachpraxis der deutschen Sprache für Studenten des ersten Studienjahres (von November 2000 bis Januar 2001) weckte mein großes Interesse für diese Methode und ihre Formen.

Zur Optimierung des didaktischen Prozesses können meines Erachtens folgende Aspekte beitragen, die ich als Elemente der Subjektivität der Lerner im didaktischen Prozess betrachte. Zu denen gehören:

\section{Physische, psychische und emotionale Sicherheit der Lerner}

Die Schule soll meiner Ansicht nach ein Platz sein, wo sich die Lerner in jeder Hinsicht sicher fühlen. Nur ein vollständiger Komfort ermöglicht ein effizientes Lernen, fördert die Konzentration und lässt die Kreativität entfalten. In den Fällen, wo der psychische und der emotionale Komfort fehlen und nur der physische Komfort anwesend ist, sind die Lernleistungen meistens gering und die Kreativität wird nicht entwickelt.

Der bekannte Psychologe Abraham Maslow schuf eine Bedürfnispyramide. Diese Bedürfnispyramide umfasst: Bedürfnis nach physiologisch Wesentlichem Nahrung und Obdach, Bedürfnis nach Sicherheit und Schutz, Bedürfnis nach Dazugehörigkeit, Gemeinschaft und Liebe, Bedürfnis nach Anerkennung, Geltung und Prestige und Bedürfnis nach Selbstverwirklichung. In der Bedürfnispyramide müssen zuerst die niederen physiologischen Bedürfnisse erfüllt werden und erst dann kann nach den höheren Bedürfnissen gestrebt werden.

Ich vertrete den Standpunkt, wenn das Bedürfnis nach Sicherheit und Schutz und das Bedürfnis nach Anerkennung, Geltung und Prestige im Kontext des Lernens nicht erfüllt werden, ist kaum ein intellektueller Entwicklungsfortschritt eines Individuums möglich. 


\section{Stressbeseitigung}

Die Stressbeseitigung ist ein weiterer Faktor, der im didaktischen Prozess nicht mehr wegzudenken ist. Der Stress beeinträchtigt die Kreativität. In Stresssituationen können die Daten nur in das Kurzzeitgedächtnis gelangen. In der mit Angst und Stress erfüllten Lernatmosphäre wird beim Menschen das negative Stresshormon - Adrenalin ausgestoßen. Wenn die in Stresssituationen gespeicherten Informationen aus dem Gedächtnis abgerufen werden, kommt es zu einer Denkblockade.

Aus psychologischer Sicht ist Stress

ein Resultat einer individuellen Interaktion - also einer Ausgangsinteraktion der äußeren und inneren Erforderungen und Möglichkeiten für einen Menschen; der Stress entsteht, wenn es zu einer Diskrepanz zwischen den Erforderungen und Möglichkeiten komm. (Neue Enzyklopädie PWN, Warszawa: Wydawnictwo PWN, eigene Übersetzung)

Der Stress in der Schule kann zu Neurosen führen (Lozanow 1978; Leontjew 1982; Włodarski; Matczak 1992 - zit. nach: Siek-Piskozub 1995). Nach Lewicki ist die Anspannung im Lernprozess nicht völlig zu beseitigen. Wenn sie aber zu groß ist, verursacht sie eine Blockade der psychophysischen Mobilisierung, die in der starren Körperhaltung, dem Abbruch des Atmens u.ä. zum Ausdruck kommt.

\section{Fröhliche Atmosphäre}

In einer fröhlichen Atmosphäre lernt man meines Erachtens effizienter, was auf Biochemisches zurückzuführen ist. Es werden dann Endorphine ausgestoßen, die für einen positiven psychophysischen, das Lernen fördernden Zustand sorgen. Die Endorphine lassen dann Acetylocholin entstehen, das eine Festigung von neuen Informationen in verschiedenen Gehirnteilen bewirkt. In einer Stresssituation ist das Ausstoßen dieser Hormone verhindert, was das Behalten von Informationen hemmt.

\section{Gute Beziehungen}

Die Kontakte auf der Ebene - Lehrer und Lerner sollen meiner Meinung nach auf Freundlichkeit und Partnerschaft beruhen. Viele Lerner empfinden zu große Angst, was ein Zusammenwirken der beiden Seiten verhindert. Der Lehrer soll ein kluger Berater, Koordinator, der sog. Mentor für die Lerner sein. Schlechte Beziehungen können die Zusammenarbeit nicht nur beeinträchtigen, sondern sogar abbrechen. 
Damit die Beziehungen korrekt sind, soll man zuerst die Teilnehmer des didaktischen Prozesses gut kennen lernen. Von Relevanz sind die Persönlichkeit und der Lernstil der Lerner. Die interpersonalen Kontakte können in direkten Gesprächen aufgenommen werden, die durch ein unverfängliches Benehmen, Offenheit und Freundlichkeit geprägt sind.

Nach Wilczyńska steht der Lerner im Zentrum des didaktischen Modells, der Lehrer dagegen koordiniert den didaktischen Prozess. Wilczyńska postuliert die Subjektivität des Lerners und stellt fest: „Diese Eigenschaft ist ein Recht und eine Berufung des Individuums, sie kann aber nicht (wie andere Rechte) als ihre Pflicht oder Beschränkung betrachtet werden..."

Sie behauptet, „das Individuum ist eine PERSON, also ein zur aktiven und absichtlichen Steuerung der eigenen Entwicklung im Rahmen seines Selbstverwirklichungsplanes fähiges Subjekt" (eigene Übersetzung), daher postuliert sie die Autonomisierung.

Pfeiffer bezeichnet den Lehrer als Schöpfer der glottodidaktischen Prozesse und zu seinen Hauptfunktionen zählt er: Organisation, Schöpfung, Steuerung und Kontrolle der glottodidaktischen Prozesse, außerdem eine Funktion der interpretierenden Person der fremdsprachlichen Kultur und eine erzieherische Funktion.

In der Suggestopädie ist der Lehrer eine Autorität, es tritt da kein Authoritarismus auf. Der Lehrer ist eine Autorität nicht wegen der Funktion, die ihm zufällt, sondern wegen seines umfangreichen Wissens, seiner Professionalität und Fähigkeit mit anderen Menschen umzugehen (Anisimowicz 2000:90).

\section{Optimismus und Selbstvertrauen}

Eine wichtige Rolle spielen Optimismus und Selbstvertrauen. Wenn die Lerner ein niedriges Selbstwertgefühl und eine negative Einstellung haben, hat der Lehrer meiner Ansicht nach Optimismus und Selbstvertrauen bei ihnen zu entwickeln. Beim didaktischen Prozess, der in bestimmten Phasen sehr kompliziert sein kann, ist seine Fortsetzung ohne die oben genannten Elemente nicht möglich. Das erste Prinzip der Suggestopädie betont das Selbstvertrauen durch die in der ersten Etappe erlangte Zufriedenheit, konzentrierte Psychoentspannung und Beseitigung von Anspannung (Łozanow 1978:259-261, zit. nach: Lewicki 1990:8).

\section{Fehler als unvermeidlicher „Feind“ des Handelns}

Ich gehe davon aus, dass Fehler als begleitendes Element beim Gelangen zur Perfektion, als etwas, was nur auf dem Weg zum Ziel steht, zu behandeln sind. In jedes menschliche Handeln sind Misserfolge, Fehler und Versehen einkalkuliert, und die Aufgabe des Menschen ist es sie zu bewältigen und zu beseitigen. 
Eine Korrektur von zahlreichen Fehlern in den aus vielen Sätzen bestehenden Aussagen soll während des Sprechens nicht erfolgen, sondern nach dessen Abschluss, obwohl das für den Lehrer viel anstrengender wegen des Merkens sein kann: eine Fehlerkorrektur ist mit einer kurzen Reihe der Übungen zum Material, in dem die meisten Fehler begangen wurden, zu verbinden. (Valette 1973, zit. nach: Komorowska 1978:212; eigene Übersetzung)

Es soll das Verhältnis zum begangenen Fehler geändert werden und ein Fehler soll als ein natürliches Element des Spracherwerbsprozesses, und nicht als ein gefährliches Symptom der Unrichtigkeit beim Verlaufen jener Prozesse betrachtet werden. (Grucza 1978, zit. nach: Komorowska 1978:212; eigene Übersetzung)

In den alternativen Methoden wird auf das sofortige Korrigieren verzichtet. Fehler werden nicht als bad habits angesehen und ihr Erscheinen lässt sich im Rahmen der psycholinguistischen Forschungen erklären und begründen (nativistische Theorie des Spracherwerbs, Interlanguage Hypothesen). Fehler verschwinden mit der Entwicklung des Lernprozesses. Die Aufgabe des Lehrers besteht darin, ständig für den richtigen Input zu sorgen: Korrigieren der Fehler erfolgt einigermaßen gelegentlich, nicht absichtlich und auf keinen Fall durch Tadeln. (zit. nach: Anisimowicz 2000:40)

Ich vertrete die Ansicht, dass Fehler während des Sprechens diskret zu korrigieren sind, gewissermaßen nebenbei, weil ihre ostentative Verbesserung zu Hemmungen beim Sprechen führen kann, und im Endeffekt zum Verlust der sprachlichen Flüssigkeit und zum sog. Stocken.

\section{Loben und Motivation}

Im didaktischen Prozess ist die Aufmerksamkeit auch auf das Loben und die Motivation zu richten. Von besonders großer Bedeutung ist es bei Anfängern. Jede kleine Leistung soll mit dem Loben belohnt werden. Fehlt dieses Element, können sich insbesondere Personen, die Schwierigkeiten mit dem Spracherwerb haben, zurückziehen und eine sprachliche Niederlage erleiden. Das Erleben des Erfolges bildet einen der wichtigsten Antistressfaktoren (Lehmann 1986:12, zit. nach: Anisimowicz 2000:98). Die Motivation beeinflusst das Gedächtnis, weil sie das Aktivierungsniveau steigert, auf die Auswahl der Reize wirkt und das Handeln präzisiert. Die Motivation löst eine Kettenreaktion aus, die nach Szałek (1992:81, zit, nach: Pfeiffer 2001) folgendermaßen aussieht:

Motivation > Auslösen von positiven Emotionen > richtige Anregung des Körpers $>$ gesteigerte geistige Aktivität $>$ Beschleunigung und Verbesserung des Spracherwerbsprozesses.

Nach Pfeiffer ist die Motivation ein System und eine Struktur von verschiedenen Motiven. Das Motiv dagegen ist ein bewusstes Handeln zur Erfüllung eines Bedürfnisses oder zum Erreichen eines Zieles. 


\section{Dynamik der didaktischen Interaktion}

Die Dynamik der didaktischen Interaktion beruht meines Erachtens auf Handeln, Stimulieren und Beseitigung von Monotonie und Ermüdung. Der Lehrer soll den didaktischen Prozess attraktiver machen, indem er Überraschungselemente einführt und Sprachsituationen zur Absurdität macht. Ein prominentes Beispiel dafür, das sich auf ,ad absurdum” bezog, beobachtete ich, als ich an den Lehrveranstaltungen von Frau Prof. Prokop am Lehrstuhl für Glottodidaktik und Translatorik im Diplomstudium im Studienjahr 2000/2001 teilnahm. Die Personen spielten Rollen der Satzteile und ihre Aufgabe war es, einen Platz in der Reihe (im Satz) zu nehmen, um die Wortfolge korrekt zu gestalten. Es wurde vor allen Dingen der Nebensatz geübt, der den Lernenden besonders große Schwierigkeiten bereitet. Die Simulation verlief bei Musik und rhythmischen Bewegungen, was alle Teilnehmer positiv angenommen haben. Sie erinnerte auf keinen Fall an eine monotone und wenig effiziente Erklärung der grammatischen Regeln.

Der Einsatz der oben genannten Elemente ist durch das suggestopädische Wirken möglich. Dieses Wirken beruht auf Musik, Entspannungstechniken, Spielen, Simulationen und äußerer Ästhetik. Die Suggestopädie trägt zur Synergie des Geistes bei. Damit diese Synergie, also das Zusammenwirken aller Funktionen des Gehirns möglich ist, soll man das Gehirn mit diversen Mitteln anregen, wie z.B. Bildern, Geräuschen und sogar Duften. Auch Farben spielen eine wichtige Rolle, weil sie sich auf die sprachliche Vorstellungskraft auswirken. Die Macht der Suggestopädie ist die Suggestie, durch die positive Emotionen und Gefühle ausgelöst werden, die ein positives Handeln initiieren. Eines der vom Vater der Suggestopädie Georgij Łozanow eingeführten Elemente ist Musik. Musik hat eine ungewöhnliche Macht und beschleunigt das Lernen. Durch Musik und entsprechende Entspannungsübungen kann unser Gehirn in den Alphazustand gebracht werden. Das ist ein Zustand der Entspannung, aber gleichzeitig der Bereitschaft. Man ist dann entspannt, aber auch konzentriert, daher ist unser Gehirn in diesem Zustand am meisten aufnahmefähig. Alphawellen treten erst im Zustand tiefer Entspannung auf (mit einer Frequenz zwischen 8 und $12 \mathrm{~Hz}$ ).

Beim Lernen der Fremdsprachen wird aus suggestopädischer Sicht die Barockmusik empfohlen. Von Bedeutung ist eine Verschmelzung der richtigen Musikarten zu einem Ganzen, dessen Teile das aktive und das passive Konzert bilden, die von Georgij Łozanow geschaffen wurden. Die Mittel, die die Suggestopädie (Łozanow 1978:261-262, zit. nach: Lewicki 1990:7) einsetzt, haben folgenden Charakter:

1. psychologischen - Anwendung von emotionalen Reizen, die zur peripheren Wahrnehmung führen, und auch Aktivierung von unbewussten Prozessen, die bewusste Tätigkeiten immer begleiten. 
2. didaktischen - Verallgemeinerung von Bedeutungen, die die Limite des Frischgedächtnisses vermeiden und somit Portionen des Materials zur einmaligen Verarbeitung vergrößern lassen.

3. künstlerischen - Einführung von besonderen Entspannungs- und Anregungsmitteln (z.B. Musik, Literatur, Rollenspiel) in den didaktischen Prozess, die zur Schaffung einer freundlichen Lematmosphäre beitragen. Lozanow führte drei Hauptprinzipien der Suggestopädie an:

- Freude, Abwesenheit von Anspannung und konzentrative Psychoentspannung

- Einheit von bewusst-unbewussten und integrativen Gehirnaktivitäten

口 Suggestive Verbindungen auf der Ebene des Reservekomplexes.

Łozanow bezog sich auf den Placeboeffekt, der früher in der Medizin eingesetzt wurde. Beim Placeboeffekt geht es um eine tatsächliche Verbesserung des Gesundheitszustands des Patienten nach Einannahme eines Pseudomedikamentes, das vom Aussehen, Geschmack u.ä. her einer echten Arznei ähnlich ist. Der Patient wird darüber nicht informiert, der Arzt dagegen verstärkt absichtlich und bewusst seinen Glauben an die heilende Wirkung des eingenommenen Medikamentes, was auch die Belastbarkeit des Patienten steigert.

Die Beziehungen auf der Ebene - Lehrer und Lerner stehen mit der Suggestie im engen Zusammenhang. Die an die Lerner vom „Mentor” gerichtete Suggestie kann meines Erachtens eine ungewöhnliche Wirkung haben. Es müssen aber gewisse Bedingungen erfüllt werden, und zwar der Lehrer muss über ein hervorragendes Fachwissen verfügen und gleichzeitig ein Partner im didaktischen Prozess sein, auch dann, wenn die Lerner den ersten Schritt auf einem Gebiet machen, oder wenn Kinder am Lernprozess teilnehmen. Jede Person erwartet Respekt und Hochschätzung.

In der Suggestopädie kommen psychische und emotionale Elemente zum Ausdruck, die einen Hintergrund für die zwischenmenschlichen Interaktionen bilden, was in den traditionellen Methoden, ausgenommen der kommunikativen Didaktik, überhaupt nicht berührt wird. Die größten Reserven sind in der Psyche des Menschen. Ein richtig und ständig stimulierter Geist nutzt in hohem Maße das schlummernde Potential. Vor Beginn des Unterrichts soll man nach Dryden und Vos den Geist reinigen, damit er „offen“ bleibt. Das kann auf vielen Wegen erreicht werden. Außer Musik und Entspannungstechniken kann man die Fantasie auch anders anregen, z.B. durch Erinnerungen an die Momente des Triumphs im Leben, eine kurze Unterhaltung mit einer anderen Person zum Lieblingsthema, oder einfach durch Gespräche über Leichtes, Farbenfrohes und Optimistisches. Die Reinigung soll die spätere Konzentration und Ablenkung von äußeren Reizen fördern.

Der Duft spielt eine kleinere Rolle als Musik, dennoch kann er sich auch auf unser Allgemeinbefinden auswirken. Nach Dryden und Vos ist es beim Merken am wichtigsten, dass „eine Assoziation eine gewisse „emotionale Ladung“ mit 
sich bringt, denn das „Filter“ in unserem Gehirn, das die Informationen in das Langzeitgedächtnis weiterleitet, ist sehr eng mit den Gehirnzentren verbunden. Darüber hinaus sollen Assoziationen möglichst viele Sinne beanspruchen: Sehkraft, Gehör, Geruchssinn, Tastsinn und Geschmack“ (eigene Übersetzung). Kurcz behauptet, das die Funktionen der beiden Hemisphären folgend sind:

- die linke Hemisphäre: analytische und beziehungsbezogene Funktionen (Vergleichen, Bestimmung von Verhältnissen zwischen Sachen und Erscheinungen), Zeitwahrnehmung (innere Uhr);

- die rechte Hemisphäre: globale, vollständige Funktionen, Wahrnehmung von optischen und räumlichen Informationen, Erkennen von Gesichtern, Gefühlen, insbesondere negativen Gefühlen, verschiedenen Melodien und Prosodie des Sprechens.

$\mathrm{Zu}$ den Hauptvoraussetzungen der alternativen Methoden gehört auch die Behauptung, die vom Wechselspiel der beiden Hemisphären handelt:

Die Sprache ist nach Anisimowicz nicht nur die Domäne der linken Hemisphäre. Die synthetischen Denk- und Lernstrategien sind für den Erwerbsprozess der Sprache so wichtig wie die analytischen Strategien. Die synthetischen Strategien werden durch den Einsatz von Musik, Symbolik der Körpersprache und ästhetischer Umgebung aktiviert.

Nach Lewicki bedeutet die Suggestopädie für den Lehrer eine Freude am Beruf, und für den Lerner eine Leichtigkeit beim Beherrschen einer Fremdsprache. Die Suggestopädie als eine von wenigen Methoden beruicksichtigt nach Anisimowicz so umfassend neurophysiologische, psychologische und soziale Hauptaspekte des Lernens und deshalb ist sie ein überzeugendes und weitsichtiges Beispiels des Lernens für einen geistig würdigen, freien Menschen.

Das suggestopädische Lehren kann meiner Ansicht nach verstärkt werden, indem die Technik - Mindmapping eingesetzt wird. Diese Technik wurde von Tony Buzon und Barry Buzon entwickelt und basiert auf einer Visualisierung von Gedanken. Das ist eine äußerst erfolgreiche Technik, denn sie spiegelt unser Gehirn wider, das aus Dendritnetzen besteht, die unlinear verbunden sind und Verzweigungen und Verästelungen bilden. Daraus resultiert, dass ein linearer Charakter von Notizen nicht effektiv ist. Im Zentrum jedes Mindmaps steht ein Schlüsselwort, von dem sich die Hauptassoziationen verzweigen. Von den Hauptassoziationen dagegen lassen sich die weiteren ableiten und von den weiteren die nächsten usw., man hat eigentlich mit der Endlosigkeit von Gedanken zu tun. Nach Möglichkeit werden Begriffe durch Bilder ersetzt. Das Mindmapping umfasst meistens folgende Phasen:

- Schreiben des Oberbegriffes und Zeichnen seiner Verzweigungen, ein Gedankenchaos ist zulässig, denn Spontaneität und Gedankenfreiheit sind von Vorteil,

- Inkubation - Zeit für Erholung und Ablenkung vom Mindmap,

- Durchsicht und Analyse des Mindmaps,

- eventuelle Einfügung weiterer Begriffe und Verbindung mit dem Ausgangsmindmap. 
Das Mindmapping kann als Gedächtnisstütze in vielen Bereichen verwendet werden, z.B. bei der Vorbereitung von Präsentationen, Prüfungsmaterial, Interviews, Vorträgen, Referaten, Seminaren, Zusammenfassungen der Bücher, Filme, Artikel, beim Notieren und u.ă.

Das Mindmapping mit Bildern ist bestimmt eine effektive Technik, das bestätigt meines Erachtens der Bildkommunikationseffekt (Eng. pictorial superiority effect). Der Bildkommunikationseffekt beruht auf der Überlegenheitswirkung bildlicher Stimuli im Vergleich zu den textlichen Stimuli. Darüber hinaus ist das Mindmapping mit dem Assoziationismus eng verbunden. Der Assoziationismus war eine schon in der antiken Philosophie und Psychologie bekannte Richtung, nach der der grundlegende Lern- und Gedächtnismechanismus auf die Entstehung von Assoziationen zurückzuführen ist.

Beim effizienten Lernen sind Entspannungstechniken anzuwenden. Das autogene Training und die progressive Entspannung können zur Verbesserung von Leistungen beitragen. In der Suggestopädie wird das autogene Training von Schultz eingesetzt.

Die Effizienz der Entspannungstechniken ist durch ihre regelmäßige Anwendung möglich. Während der Übungen soll man Muskeln lockern, richtig ein- und ausatmen und Gedanken beruhigen. Die Entspannungstechniken lassen den Stress abbauen, fördern die Konzentration und verbessern den psychophysischen Zustand.

Äußere Ästhetik ist ein weiterer relevanter Faktor im didaktischen Prozess. Von Bedeutung ist die Sorge für Unterrichtsräume. Sie sollen die Fantasie anregen und den ästhetischen Erwartungen der Teilnehmer des didaktischen Prozesses entsprechen. Zur Anregung der Fantasie können nach Dryden und Vos verschiedene Plakate dienen, wo Farben und Bilder vorherrschen. Blumen können auch ein Verschönerungselement für Unterrichtsräume sein, wo Assoziationen, Vorstellungskraft und Kreativität entwickelt werden. Im Falle der korrekt geführten Lehrveranstaltungen ist meiner Ansicht nach auch auf scheinbar triviale Dinge zu achten, die jedoch auch von Bedeutung sind, z.B. eine richtige Aufstellung von Bänken. Sie sollen nicht nacheinander stehen, sondern eine geschlossene geometrische Figur bilden - Halbkreis. Dadurch kann man den Blickkontakt halten und die Kommunikation leichter gestalten. Außerdem sitzen oft schüchterne Personen in hinteren Bänken, was durch diese Lösung zu vermeiden ist.

Spiele und Simulationen: Die ludischen Strategien spielen beim Unterrichten eine relevante Rolle. Schon 1880 postulierte Gouin das Lernen einer Sprache in Form von Spiel und Bewegung (Neuner 1987:148, zit. nach: Anisimowicz 2000:22). Auf Grund meiner Erfahrungen kann ich feststellen, dass Spiele und Simulationen Stress und Hemmungen beseitigen, das Lernen abwechslungsreicher 
machen, den Stoff besser aneignen lassen und positive Emotionen liefern, die den Lernprozess beschleunigen. Ich vertrete denselben Standpunkt wie die Pädagogen, die in Polen in diesem Bereich viele Forschungen durchgeführt haben, z.B.: Filipowicz, Golankiewicz, Kamola und andere. Durch Spiele entsteht ein psychologisches Klima und in einem solchen Klima kommen nach Siek-Piskozub solche Charaktereigenschaften zum Ausdruck, wie Temperament, Quellen und Dynamik der Motivation, Leistungsdrang, Denkstil, Risikobereitschaft, Konfliktanfälligkeit, Adäquatheit der Selbstbewertung, Extravertiertheit und Introvertiertheit. Auf diesem Wege können interpersonale Kontakte aufgenommen werden, die sich meiner Meinung nach in hohem Maße auf die Qualität des didaktischen Prozesses auswirken.

Spiele sollen ein festes Element des Unterrichts sein, besonders gilt das für Kinder, denn das Spiel ist nach Siek-Piskozub für Kinder ein instinktives Bedürfnis (Trieb), dessen Erfüllung zur Entwicklung ihrer physischen und intellektuellen Ressourcen beiträgt.

Ich vertrete die Auffassung, dass auch Jugendliche und Erwachsene verschiedene ludische Formen bevorzugen. Das ist wohl auch darauf zurückzuführen, was Lozanow festgestellt hat: „Manche brauchen sich sogar hinter einer Schutzmaske zu verstecken, damit ihr Selbstwertgefühl bei schwierigen Kommunikationsversuchen in der Fremdsprache nicht in Mitleidenschaft gezogen wird" (eigene Übersetzung).

Homo sapiens ist von Natur aus homo ludens. Das Gehim soll meiner Ansicht nach durch alles Mögliche stimuliert werden. Die Stimulierung soll durch Bild, Wort, Kontrast, Duft, Lachen, Scherz, Absurdität, Ernst, Geste, Mimik, Ärger, Freude, Nachdenklichkeit, Emotion, Musik, Spiel, Simulation, Assoziation und ein anderes Gehirn erfolgen. Alle oben genannten Elemente korrelieren miteinander und ergänzen einander. Ich gehe davon aus, dass die Synergie des Geistes ohne Berücksichtigung der Elemente der Subjektivität, wie das richtige Innere, partnerschaftliche Beziehungen, fröhliche Atmosphäre oder Optimismus nicht möglich ist. Es ist auch das Gleichgewicht zwischen dem psychischen und dem physischen $\mathrm{Zu}-$ stand von Bedeutung. Damit das Gehirn effizienter arbeitet, soll man auf die Gesundheit achten. Nach Dryden und Vos ist eine wertvolle Diät folgend:

- $\quad$ üppiges Frühstück mit vielen frischen Obstsorten

- herzhaftes zweites Frühstück, im besten Fall mit Salat aus frischem Gemüse

- Fisch- und Pflanzenfette als unabdingbares Diätelement

- regelmäßige Übungen zur Lieferung von Sauerstoff in das Gehirn

- Entfernung von Toxinen aus dem Organismus.

Die Harmonie zwischen dem psychischen und dem physischen Zustand ist auch eine Voraussetzung für ein effektives Funktionieren des Gehirns.

Ein guter Lehrer ist meiner Ansicht nach ein guter „Psychologe“. Er erkennt und definiert Bedürfnisse, Erwartungen und Arbeitsstile der Lerner. Zu diesem Zweck können verschiedene von Gardner beschriebene Intelligenzarten dienen. 
Nach Gardner existieren folgende Intelligenzarten (Dryden, Vos 2000:121):

- sprachliche Intelligenz

- logische oder mathematische Intelligenz

- musikalische Intelligenz

- räumliche und visuelle Intelligenz

- kienästhetische Intelligenz

- interpersonale Intelligenz

- intrapersonale Intelligenz

- naturwissenschaftliche Intelligenz.

Daniel Goleman führte zudem den Begriff der emotionalen Intelligenz ein. Beim Lehren der Fremdsprachen ist es wichtig, bei den Lernern eine Intelligenzart zu definieren, um den Unterricht korrekt zu gestalten und somit den Lernern die Möglichkeit zu geben, eine Intelligenzart oder mehrere Intelligenzarten zu präsentieren und weiter zu entfalten. Im besten Fall verfügen die Lerner über die sprachliche Intelligenz. Fehlt sie aber im Lernprozess, können andere Wege gefunden werden, z.B. musikalisch begabte Personen sollen völlig die musikalische Intelligenz nutzen, visualisierungsorientierte Personen - die visuelle und räumliche Intelligenz, kienästhetische Lerner dagegen die kienästhetische Intelligenz. Die musikalische, visuelle und räumliche Intelligenz kann bei Inszenierungen im Fremdsprachenunterricht in Anspruch genommen werden. Die Personen mit der musikalischen Intelligenz können einen Beitrag in diesem Bereich leisten. Diejenigen, die über die visuelle und räumliche Intelligenz verfügen, können sich mit der Bühnenbildung und Choreographie befassen.

Die Personen, die die logische Intelligenz haben, können sie z.B. beim Aneignen der grammatischen Regeln nutzen. Die interpersonale Intelligenz kann bei sprachlichen Projekten von Vorteil sein, wo viele Personen tätig sind. Ich vertrete die Auffassung, das die interpersonale Intelligenz von besonderer Relevanz ist, denn die Personen, die in der Muttersprache Kontakte ohne Schwierigkeiten aufnehmen, können diese Fähigkeit auf die Plattform der Fremdsprache übertragen. Das prominenteste Beispiel für die interpersonale Intelligenz ist Adolf Hitler, der so viele Menschen zu gewinnen vermochte, wie niemand in der menschlichen Geschichte. Ich stehe auf dem Standpunkt, dass über diese Intelligenz jeder gute Lehrer verfügen sollte, weil die Kommunikationsebene, die dadurch entstehen kann, ermöglicht eine richtige didaktische Zusammenarbeit. Zwischen den Teilnehmern des didaktischen Prozesses wird dann ein Kontakt geknüpft, der einen Ausgangspunkt für die bilaterale Kooperation bildet. Die intrapersonale Intelligenz ist einigermaßen eine Introspektion, eine Vertiefung des eigenen Ego, und eben das ist eine Basis für ein Handeln, hier für das Fremdsprachenlernen. Die naturwissenschaftliche Intelligenz, die ein umweltfreundlicher Lebensstil bedeutet, kann eine Grundlage für Harmonie und innere Ruhe bilden, was im ganzen Lernprozess auch von großer Bedeutung ist. 
Die emotionale Intelligenz beruht auf einer richtigen Steuerung von eigenen Emotionen, Eifer, Temperament, Empathieentwicklung und entsprechenden sozialen Verhaltensweisen. Nach Goleman hängt der Erfolg des Menschen vom Koeffizient der emotionalen Intelligenz ab. Meines Erachtens liegt die Qualität des didaktischen Prozesses auch an der emotionalen Intelligenz, denn die richtige Steuerung von Emotionen und das korrekte Vorgehen des Lehrers garantiert gute interpersonale Kontakte zwischen allen Teilnehmern des didaktischen Prozesses. Die emotionale Intelligenz ist eng mit der interpersonalen Intelligenz verknüpft.

Nach der Analyse der Subjektivität und ihrer Elemente im didaktischen Prozess komme ich zu dem Schluss, dass dieser Prozess nur dann effektiv sein kann, wenn der Einsatz seiner einzelnen Bestandteile global ist, weil sie auf einem Wechselspiel basieren. Man kann daher z.B. gute Beziehungen von Sicherheit, oder Spielen und Simulationen, oder fröhlicher Atmosphäre nicht trennen.

Die Grenzen zwischen ihnen sind fließend und der didaktische Erfolg ohne holistische Betrachtung der oben genannten Elemente ist nicht möglich. Die Lerner, die über mehrere von den oben geschilderten Intelligenzarten verfügen (im Idealfall - über alle), können den didaktischen Prozess selbst gestalten, der ihnen den Weg zur Selbstverwirklichung öffnet. Meines Erachtens kann die Berücksichtigung dieser grundlegenden Bedingungen den didaktischen Prozess in hohem Maße optimieren.

Der didaktische Prozess ist meiner Ansicht nach ein System von Bestandteilen, die aufeinander wirken. Die Isolierung mancher Komponenten von anderen kann zu Störungen beim Funktionieren oder sogar zum Kollaps dieses Systems führen. Das suggestopädische Lehren ist ein Lehren der Zukunft, weil es die emotionale und psychische Seite des Menschen in Betracht zieht, was in den traditionellen Lernmethoden tabu war. Die Suggestopädie weckt die schlummernden Reserven des menschlichen Geistes.

\section{BIBLIOGRAFIE}

Anisimowicz, B. (2000): Alternatywne nauczanie języków obcych w XX wieku. Sugestopedia. Warszawa: Wydawnictwo DIG.

Buzan, T.; Buzan, B. (1993): Mapy myśli. Lódź: Wydawnictwo „Ravi”.

Dryden, G.; Vos, Jeannette (2000): Rewolucja w uczeniu. Poznań: Wydawnictwo Moderski i S-ka.

Edelmann, W. (1988): Suggestopädie/Superlearning. Ganzheitliches Lernen - das Lernen der Zukunft? Heidelberg: Roland Asanger Verlag.

Goleman, D. (1997): Inteligencja emocjonalna. Poznań: Media Rodzina of Poznań.

Hinkelmann, K.G. (1986): Suggestopädie. Bremen: PLS Psychologische Lernsysteme, Privatlehrinstitut und Verlag.

Komorowska, H. (1978): Sukces $i$ niepowodzenie w nauce języka obcego. Warszawa: Wydawnictwa Szkolne i Pedagogiczne. 
Kubiak, P. (2000): Suggestopädie in Theorie und Praxis - Versuch einer synoptischen Bestandsaufnahme. Unveroff. Magisterarbeit. Katedra Glottodydaktyki i Translatoryki UAM, Poznań.

Kurcz, I. (2000): Psychologia jezzka i komunikacji. Warszawa: Wydawnictwo Scholar.

Langen, D. (1999): Autogenes Training. München: Gräfe und Unzer Verlag GmbH.

Lewicki, E.R. (1990): Innowacyjność w sugestopedycznym nauczaniu języków obcych. Wrocław: Wydawnictwo Uniwersyteckie.

Mizerski, S. (2001): Nowa Szkoła. Polityka, Nr. 3 (2281), 20.01.2001.

Olejnik, K. (2001): Psychologowie stali się sprzymierzeńcami gwiazd sportu. Wprost, Nr. 3 , 21.01.2001.

Pfeiffer, W. (2001): Nauka jezyków obcych. Od praktyki do praktyki. Poznań: Wydawnictwo Wagros.

Pöppel, E.; Edingshaus, A.-L. (1998): Mózg-tajemniczy kosmos. Warszawa: Państwowy Instytut Wydawniczy.

Prokop, I. (1999/2000): Sugestopedia. Audiovisuelle Medien. Katedra Glottodydaktyki i Translatoryki UAM, Poznan.

Siek-Piskozub, T. (1995): Gry, zabawy i symulacje w procesie glottodydaktycznym. Poznań: Wydawnictwo Naukowe UAM.

Skudrzykowa, A.; Urban K. (2000): Maty stownik terminów z zakresu socjolingwistyki i pragmatyki językowej. Warszawa: Spólka Wydawniczo-Księgarska.

Wilczyńska, W. (1999): Uczyć się czy być nauczanym. O autonomii w przyswajaniu języka obcego. Warszawa: Wydawnictwo PWN S.A.

www.bwl.uni-mannheim

www.rhetorik.ch

www.thur.de 